Annales Geophysicae (2002) 20: 213-223 (C) European Geophysical Society 2002

(5)

\title{
Aspect sensitivity measurements of polar mesosphere summer echoes using coherent radar imaging
}

\author{
P. B. Chilson ${ }^{1,2}$, T.-Y. Yu ${ }^{3,4}$, R. D. Palmer ${ }^{3}$, and S. Kirkwood ${ }^{1}$ \\ ${ }^{1}$ MRI Atmospheric Research Programme, Swedish Institute of Space Physics, Kiruna, Sweden \\ ${ }^{2}$ Now at Cooperative Institute for Research in Environmental Sciences (CIRES), University of Colorado / NOAA, and \\ Environmental Technology Laboratory, National Atmospheric and Oceanic Administration, Boulder, CO, USA \\ ${ }^{3}$ Department of Electrical Engineering and Center for Electro-Optics, University of Nebraska, Lincoln, NE, USA \\ ${ }^{4}$ Now at National Center for Atmospheric Research, Boulder, CO, USA
}

Received: 13 February 2001 - Revised: 2 August 2001 - Accepted: 28 August 2001

\begin{abstract}
The Esrange VHF radar (ESRAD), located in northern Sweden $\left(67.88^{\circ} \mathrm{N}, 21.10^{\circ} \mathrm{E}\right)$, has been used to investigate polar mesosphere summer echoes (PMSE). During July and August of 1998, coherent radar imaging (CRI) was used to study the dynamic evolution of PMSE with high temporal and spatial resolution. A CRI analysis provides an estimate of the angular brightness distribution within the radar's probing volume. The brightness distribution is directly related to the radar reflectivity. Consequently, these data are used to investigate the aspect sensitivity of PMSE. In addition to the CRI analysis, the full correlation analysis (FCA) is used to derive estimates of the prevailing three-dimensional wind associated with the observed PMSE. It is shown that regions within the PMSE with enhanced aspect sensitivity have a correspondingly high signal-to-noise ratio (SNR). Although this relationship has been investigated in the past, the present study allows for an estimation of the aspect sensitivity independent of the assumed scattering models and avoids the complications of comparing echo strengths from vertical and off-vertical beams over large horizontal separations, as in the Doppler Beam Swinging (DBS) method. Regions of enhanced aspect sensitivity were additionally shown to correlate with the wave-perturbation induced downward motions of air parcels embedded in the PMSE.
\end{abstract}

Key words. Ionosphere (polar ionosphere) Meteorology and Atmospheric Dynamics (middle atmosphere dynamics) Radio Science (Interferometry)

\section{Introduction}

Ecklund and Balsley (1981) announced the surprising existence of layers of strong VHF backscatter originating from the northern polar mesopause region. Additionally, the echoes were shown to be present only during the summer months. Consequently, this phenomenon is referred to as po-

Correspondence to: P. B. Chilson

(Phillip.Chilson@noaa.gov) lar mesosphere summer echoes (PMSE). These radar echoes are orders of magnitude greater than expected from electron density inhomogeneities created by turbulence, and as such, they challenge the prevailing theories of radar backscatter. Since the initial report of PMSE, similar echoes have been detected at mid-latitudes (Reid et al., 1989; Thomas et al., 1992), and at Southern polar latitudes (Woodman et al., 1999). However, there is a disparity in the amount of PMSE activity observed at southern latitudes compared with northern latitudes (Balsley et al., 1995). During the ensuing years, a rich body of literature has developed in an attempt to explain PMSE, among which are several review papers (Röttger et al., 1988; Cho and Kelley, 1993; Cho and Röttger, 1997).

Certain recurring features of PMSE have been observed over the years. Many of these are outlined in the review articles mentioned above. The most obvious feature is the connection between PMSE and the cold temperatures of the polar summer mesopause region. This has led to the association of PMSE with the presence of ions and charged aerosols (e.g. Cho et al., 1992). Furthermore, it has been established that the PMSE are in some way associated with the occurrence of noctilucent clouds (NLC), which are diffuse layers of ice crystals that constitute the highest observed clouds in our atmosphere (Nussbaumer et al., 1996; von Zahn and Bremer, 1999; Stebel et al., 2000). There are many intriguing aspects of PMSE; however, here we focus on regions within the echoing layers that are aspect sensitive when probed with VHF radars. Aspect sensitivity refers to the dependence of echo power on the angle of incidence of the radio wave, with the echo power decreasing as the incident angle is increasing (Gage and Green, 1978; Röttger and Vincent, 1978; Hocking, 1987; Hocking et al., 1989).

Early on, it was recognized that PMSE observations at VHF exhibited aspect sensitivity (Czechowsky et al., 1988). PMSE have been broadly grouped into two categories: type I echoes, which have broad spectral widths and very little aspect sensitivity, and type II echoes, which are characterized by narrow spectral widths and are strongly aspect sensitive (Blix, 1999). In at least one study, 10\% of the PMSE were 
found to be type I and 90\% were type II (Czechowsky and Rüster, 1997; Blix, 1999). Furthermore, it has been shown that type I echoes are typically observed in the upper portions of the PMSE (Huaman and Balsley, 1998; Blix, 1999).

Aspect sensitivity of PMSE is largely attributed to Fresnel scatter or Fresnel reflection. Although Hocking and Röttger (1997) have demonstrated that large-scale gradients in the background electron density cannot account for PMSE, it is still possible for small-scale fluctuations to contribute (Blix, 1999). These could be caused, for example, by highly anisotropic scatterers at the edges of turbulent layers (Woodman and Chu, 1989). Additionally, Blix (1999) has proposed that a two-stream instability mechanism might account for PMSE, although to date, no empirical evidence has been found to support the claim.

Typically, aspect sensitivity estimates are obtained by comparing the echo strengths from vertical and off-vertical radar beams. Although this method has been used in the mesosphere for the study of PMSE, it suffers from the disadvantage of large horizontal separations of the radar sampling volumes at such heights. For example, at a height of $85 \mathrm{~km}$, the horizontal separation between a vertical beam and one directed $7^{\circ}$ off-vertical will be more than $10 \mathrm{~km}$. If opposing off-vertical beams are used, which is often the case, then the horizontal separation doubles. It is questionable whether one should assume that scatterers observed over such large horizontal separations are comparable.

Although not widely used, it is possible to make in-beam estimates of the aspect sensitivity of PMSE by using the full correlation analysis (FCA) technique (Zecha et al., 2001). By comparing data obtained from SA and DBS mode observations, Zecha et al. (2001) demonstrated that both techniques are equally suitable for studies of PMSE. Furthermore, by combining the data sets, the authors concluded that PMSE result from a scattering mechanism rather than reflection.

In the present study, coherent radar imaging (CRI) will be used. Similar to FCA, CRI enables in-beam calculations of various atmospheric parameters through the use of data from spatially separated receiving antennas. As we will discuss later in the paper, CRI is a more evolved (and computationally demanding) technique than the FCA. However, the results can be quite impressive.

We present a brief description of the experimental configuration of the radar used in this study in Sect. 2, followed by a discussion of the data analysis techniques in Sect. 3. The latter addresses the application of FCA and CRI to the PMSE data. FCA has been widely used in radar studies of the atmosphere, whereas CRI is still relatively new. Therefore, we have focused our attention on the implementation of CRI. By using FCA and CRI, it is shown that atmospheric waves play a role in establishing regions of enhanced aspect sensitivity within PMSE. It is proposed that the connection comes partially through the induced vertical atmospheric motions. Additional results from the experiment are given in $\mathrm{Yu}$ (2000) and Yu et al. (2001).

\section{Experimental configuration}

The Esrange VHF radar (ESRAD), located in northern Sweden $\left(67.88^{\circ} \mathrm{N}, 21.10^{\circ} \mathrm{E}\right)$, is well suited for the study of PMSE. Providing the ability to monitor and study PMSE was a driving factor behind the decision to initially acquire a mesosphere-troposphere-stratosphere (MST) radar in Sweden. The ESRAD system's frequency is $52 \mathrm{MHz}$, and its peak transmit power is $72 \mathrm{~kW}$. It uses a 140-element array of Yagi antennas for transmission and reception. The one-way half-power beamwidth of the radar beam is $7.8^{\circ}$. ESRAD uses six independent receivers to sample the echo power from the atmosphere. The receivers are cabled to spatially separated subarrays of the main antenna array. A more complete description of ESRAD is given in Chilson et al. (1999).

During the period of 31 July to 4 August 1998, ESRAD was operated in a mode that permits the implementation of FCA (Briggs, 1984) and CRI (Palmer et al., 1998) on the recorded data. The sampling range was $80.0-89.9 \mathrm{~km}$, with a resolution of $150 \mathrm{~m}$. A 16-bit complementary coded pulse was transmitted, and the received radar signal was stored as a 256-point complex time series for each receiver channel. For each time series point, 64 coherent integrations were performed, which resulted in a sampling period of $88.3 \mathrm{~ms}$. The radar dwell time was $22.6 \mathrm{~s}$, but when taking the time required for data transfer into consideration, $\sim 60 \mathrm{~s}$ was needed between successive dwell periods. The radar beam was directed vertically during all measurements. (See Yu (2000) and $\mathrm{Yu}$ et al. (2001) for additional information on the configuration of the radar.)

\section{Data analysis and overview}

\subsection{FCA analysis}

The current implementation of the FCA analysis is described in $\mathrm{Yu}(2000)$ and $\mathrm{Yu}$ et al. (2001). Basically, the FCA was implemented using the algorithm proposed by Briggs (1984), except some rejection criteria were modified or removed. FCA provides estimates of the signal-to-noise ratio (SNR) and the components of the three-dimensional wind field. These are presented in Fig. 1 as range-time-pseudocolor (RTP) plots. Note that only those data are shown for which the SNR was greater than or equal to $0 \mathrm{~dB}$. The time and height resolution are $60 \mathrm{~s}$ and $150 \mathrm{~m}$, respectively.

Figure 1 illustrates the complexity of the structures that are evident in the PMSE in both time and height. Two features in the RTP plot of SNR are the bifurcated regions of the layers and the layers' downward phase propagation. The bifurcated region is most evident during the time interval of 12:30-14:00 UT at heights of roughly $85-87 \mathrm{~km}$. Both of these features are commonly observed in PMSE. One explanation for the bifurcated structure is the strong turbulence produced by vertical wind shear (Hooper and Thomas, 1997). Qualitatively, indications of vertical shear can be seen in the 
RTP plots of the zonal and meridional wind components in Fig. 1. The times and heights of the shear region correspond with those of the bifurcated layers. A more quantitative treatment is given below. The downward phase propagation is related to the background field of the wind and temperature, as modulated by upward propagating gravity waves (Chilson et al., 1997). We note that the downward phase becomes more apparent when discussing estimations of tilt angles of the PMSE layers in Sects. 3.2 and 4. The short-period modulations apparent in the velocity fields are indicative of wave activity.

\subsection{Coherent radar imaging}

The underlying motivation behind CRI is the creation of angular maps of the atmospheric reflectivity field on spatial scales smaller than the angular resolution of a radar's probing pulse. The maps are generated by forming synthesized radar beams, which are selectively steered in angle within the sampling volume. To achieve this, the amplitudes and/or phases of the received signals from two or more spatially separated receivers are adjusted and then combined according to one of several different available methods. Note that CRI is different than Doppler beam swinging (DBS). In DBS, phase offsets are introduced to a collection of antenna elements for the purpose of steering the radar beam. The received signals from the different antenna elements are then combined and sent to a single receiver. Consequently, each measurement is constrained to a single beam-pointing direction. By having dedicated receivers for each antenna element (or subarray of antenna elements), there are no limits to the number of beam-pointing directions. However, the overall dimension and sub-array spacing limit the resolution, or independence of each pointing direction.

Although radar imaging techniques have been used for decades in such areas as radio astronomy and seismic research (Thompson, 1986; Haykin et al., 1985), they have only been introduced to atmospheric studies within the last ten years (Kudeki and Sürücü, 1991). Most of the applications of CRI for atmospheric studies have relied on onedimensional implementations of the technique (Kudeki and Sürücü, 1991; Hysell, 1996; Hysell and Woodman, 1997). Recently, however, Palmer et al. (1998) advanced a generalized two-dimensional framework for CRI. In particular, they focused on the Capon imaging technique, although Fourierbased imaging is also discussed. The Capon method, since it uses an optimally constrained approach that considers information contained within the signals, provides superior results to those from traditional Fourier-based methods. A simulation study in which Fourier-based, Capon, and maximum entropy methods were compared is provided by $\mathrm{Yu}$ et al. (2000).

The application of CRI to atmospheric radar signals is described in detail in Woodman (1997) and Palmer et al. (1998). Here we only provide a conceptual overview of CRI. As we have mentioned, the desired quantity is an estimate of the angular distribution of the atmospheric reflectivity.
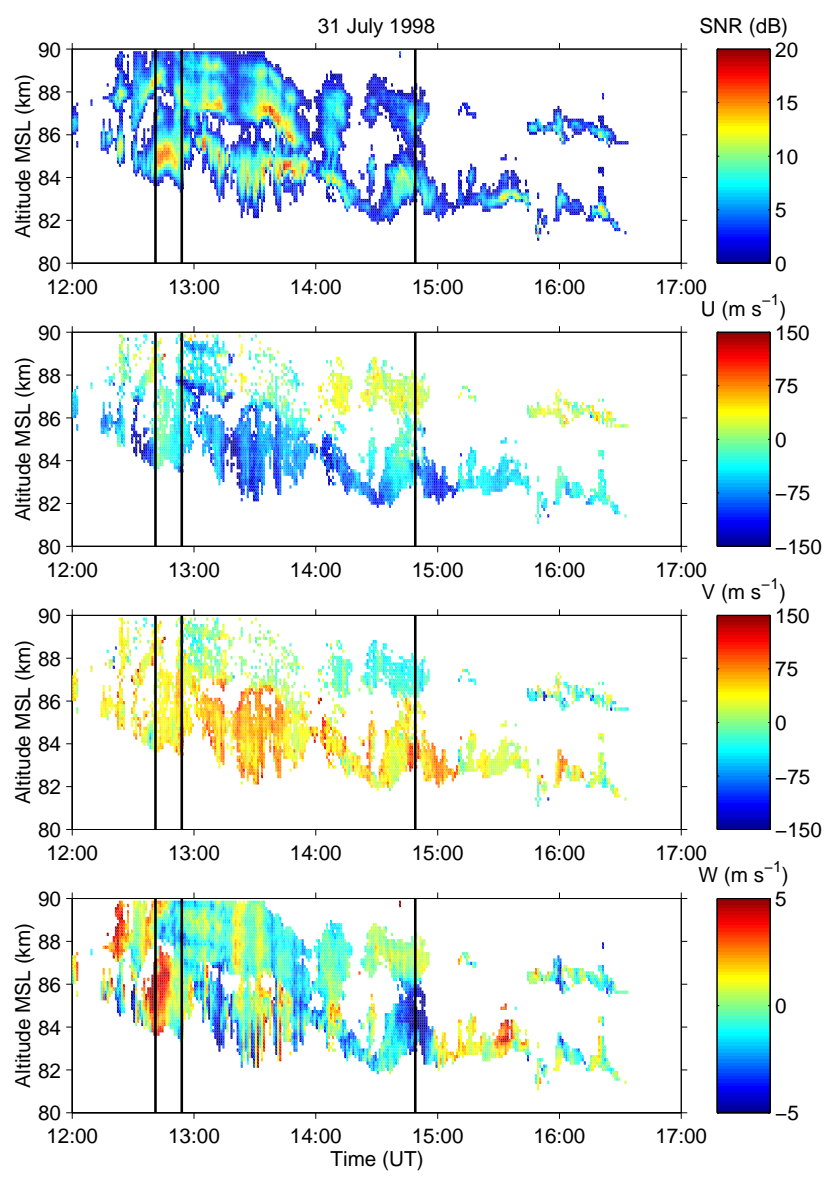

Fig. 1. Range-time pseudocolor plots of the signal-to-noise ratio (SNR); zonal (U) and meridional (V) components of the horizontal wind; and the vertical velocity $(\mathrm{W})$. The black vertical lines are used to flag times that have been selected for closer examination.

The reflectivity is proportional to the brightness, a term commonly used in optical and radio astronomy to represent the power received per unit solid area per unit frequency. When used in the context of atmospheric radar, "frequency" is directly related to the Doppler shift. In radar imaging, it is not the brightness that is measured, but rather the visibility, which is expressed in terms of cross-correlation functions or cross spectra that have been calculated from the complex time series data for the different receivers (Palmer et al., 1999; Chau and Woodman, 2001). Since visibility can be expressed as a linear superposition of weighted brightnesses, estimation of the brightness is an inversion problem.

Using CRI, estimates of the brightness distribution have been generated for those times and heights for which the SNR is greater than or equal to $0 \mathrm{~dB}$. These are then mapped onto $64 \times 64$ grids for which the range of zenith angles is from $-7^{\circ}$ to $+7^{\circ}$. Examples of such maps for a limited range of continuous time and height are shown in Fig. 2. We note that beamwidth effects are included in the brightness maps. These maps are used to study the structure of the radar reflectivity field. By locating the peak in the brightness maps, one can readily find the mean angle of arrival (MAOA) of 


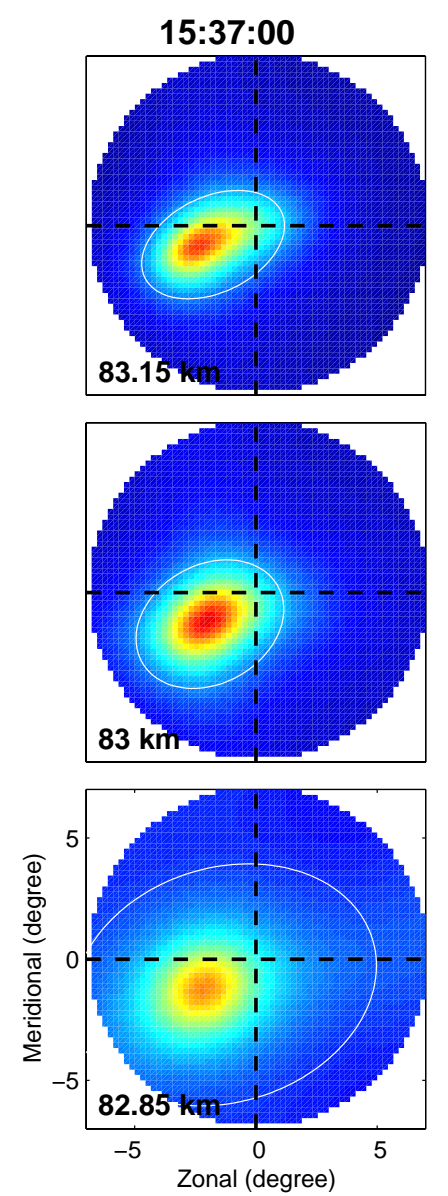

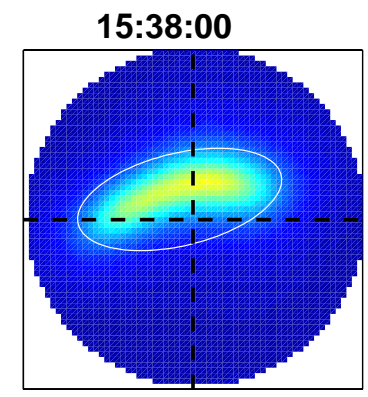
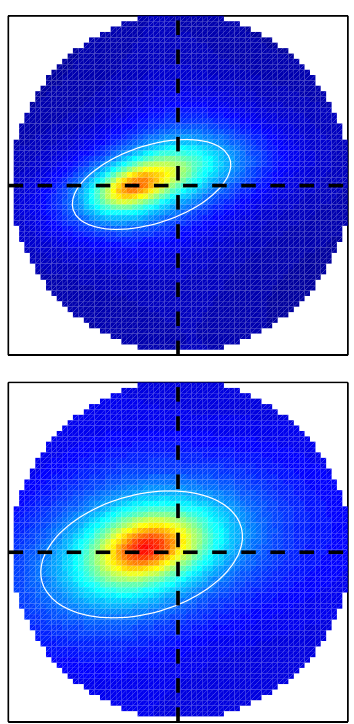

15:39:00
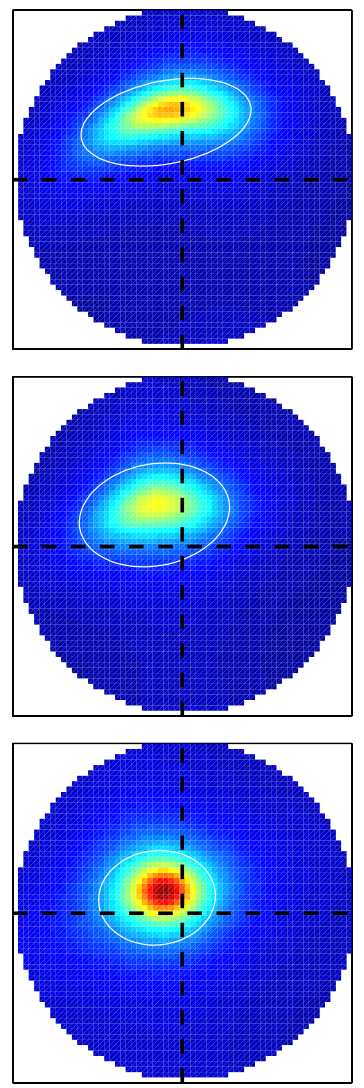

$15: 40: 00$
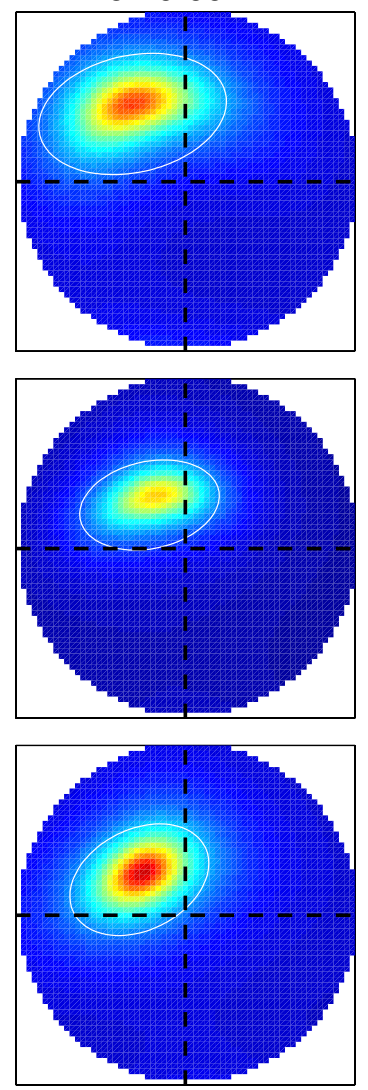

Fig. 2. Examples of the brightness distribution of PMSE that are estimated using Capon CRI. Each image shows the PMSE structure within the radar beam from $-7^{\circ}$ to $7^{\circ}$ and is estimated every $50 \mathrm{~s}$. The range of values shown in each image ranges from 0 to 1 . By applying a two-dimensional Gaussian fitting algorithm, a set of parameters $\left(\mu_{x}, \mu_{y}, \sigma_{x}, \sigma_{y}\right.$, and $\left.\rho\right)$ can be determined. The white contour lines indicate a constant value of 0.1 using the estimated Gaussian parameters.

the backscattered echo power. Furthermore, the brightness maps can be used to quantify the width of brightness distribution. We begin by assuming that the estimated brightness distribution can be described by a two-dimensional Gaussian function of the form:

$$
\begin{array}{r}
P\left(\theta_{x}, \theta_{y}\right)=\exp \left\{-\frac{1}{2(1-\rho)}\left[\frac{\left(\theta_{x}-\mu_{x}\right)^{2}}{\sigma_{x}^{2}}\right.\right. \\
\left.\left.+\frac{\left(\theta_{y}-\mu_{y}\right)^{2}}{\sigma_{y}^{2}}-\frac{2 \rho\left(\theta_{x}-\mu_{x}\right)\left(\theta_{y}-\mu_{y}\right)}{\sigma_{x}^{2} \sigma_{y}^{2}}\right]\right\},
\end{array}
$$

where $\theta_{x}$ and $\theta_{y}$ are the zonal and meridional components of the zenith angle, respectively. The location (MAOA) and half-width of the peak in the Gaussian structure in both the zonal and meridional directions are given by $\mu_{x}, \mu_{y}, \sigma_{x}$, and $\sigma_{y}$, respectively. The correlation coefficient that specifies the dependence between the zonal and meridional directions is given by $\rho$. It is assumed that the brightness distribution is unimodal. Given the assumed form of the distribution function, it is straightforward to derive estimates of $\mu_{x}, \mu_{y}, \sigma_{x}$, and $\sigma_{y}$ from the brightness maps.
Estimates of the MAOA and half-width of the brightness distribution are shown in Fig. 3. Note that $\sigma_{x}^{\prime}$ and $\sigma_{y}^{\prime}$, and not $\sigma_{x}$ and $\sigma_{y}$, are shown in the figure. The values of $\sigma_{x}^{\prime}$ and $\sigma_{y}^{\prime}$ have been calculated in a coordinate system that has been rotated so as to have its abscissa and ordinate correspond to the major and minor axes, respectively, of the brightness distribution. Consequently, the primed quantities of $\sigma_{x}^{\prime}$ and $\sigma_{y}^{\prime}$ express the half-widths of the brightness distribution along its major and minor axes, respectively. The rotation angle $\Theta$, which has a positive sense in the counterclockwise direction, is also shown in Fig. 3. For the most part, the rotation angle is close to zero, indicating that the major axes of the distributions are aligned in the zonal direction.

The data presented in Fig. 3, together with the SNR and wind estimates in Fig. 1, provide an indication of significant wave activity during the PMSE event. In particular, evidence of oscillations in the vertical extent are apparent in the RTP plots of SNR, $\mu_{x}, \mu_{y}, \sigma_{x}^{\prime}$, and $\sigma_{y}^{\prime}$. The PMSE is bifurcated into upper and lower regions, and the upper region contains additional sublayers. All of these descend in time. Since a complementary code was implemented during the observa- 

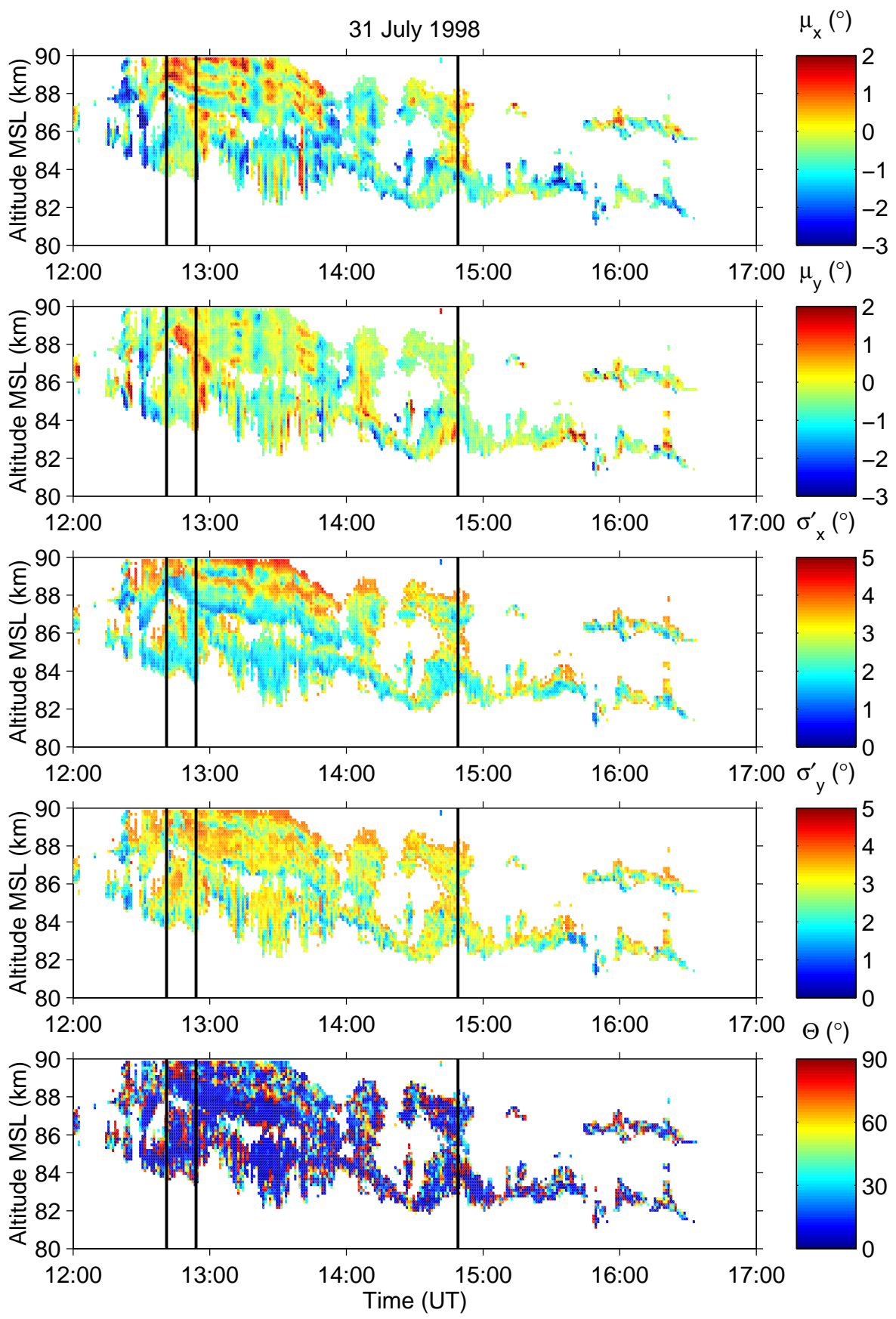

Fig. 3. Range-time pseudocolor plots of MAOA estimates $\left(\mu_{x}\right.$ and $\left.\mu_{y}\right)$; the halfwidths of the brightness distribution $\left(\sigma_{x}^{\prime}\right.$ and $\sigma_{y}^{\prime}$ ); and the rotation angle $(\Theta)$. See text for a description of these quantities. The black vertical lines are used to flag times that have been selected for closer examination. tions, we were initially suspicious that the sublayering may be an artifact of the decoding process. However, after some investigation, we concluded that the sublayers are real. Particularly striking are the descending sublayers of positive and negative MAOAs, as seen in the data for $\mu_{x}$. This feature is not as apparent in the data for $\mu_{y}$, which demonstrates that the oscillations are primarily aligned zonally. We also find descending patterns of larger and smaller values of $\sigma_{x}^{\prime}$, with a propensity for smaller $\sigma_{x}^{\prime}$ to occur near the bottom sides of the PMSE sublayers. We must ask to what extent the features seen in these data are related. We discuss this further in the next section.

\section{Aspect sensitivity and wave dynamics}

The PMSE data presented here so far suggest a connection between atmospheric waves, height profiles of SNR, and aspect sensitivity. Indeed, the interdependence between wave dynamics and the PMSE structure has a plausible foundation, as we demonstrate in this section. It should not be surprising that the occurrence and structure of the PMSE are linked to wave activity on many scales. Numerous papers have already addressed this topic, such as Fritts et al. (1988); Röttger et al. (1990); Cho and Morley (1995); Chilson et al. (1997); Kirkwood and Réchou (1998), just to name a few. 


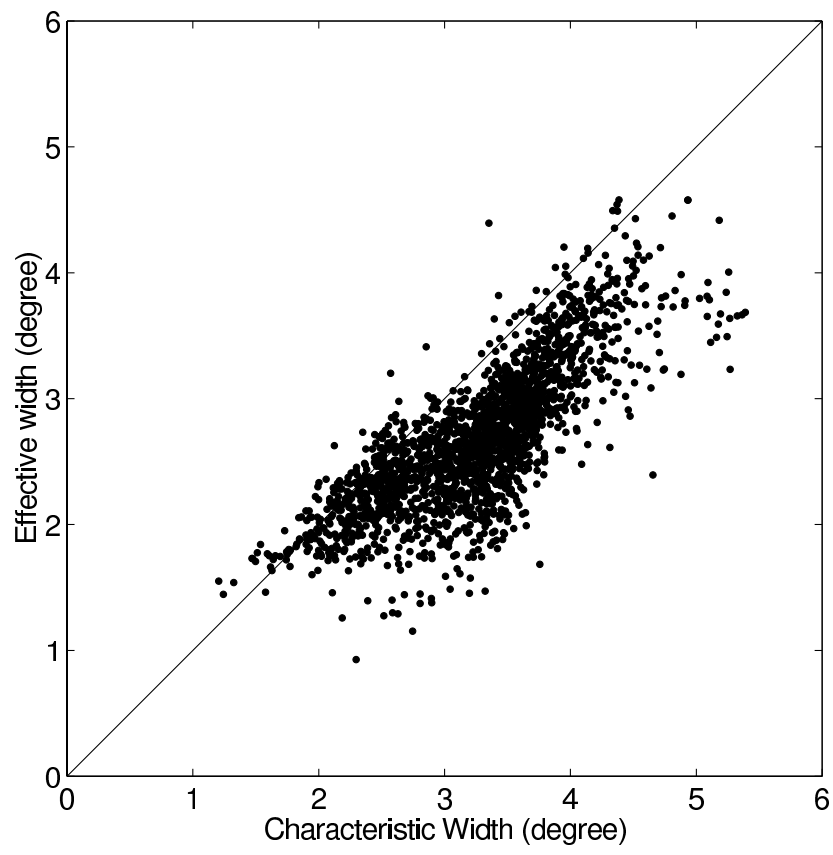

Fig. 4. Scatter diagram of the characteristic width obtained from the Capon CRI and the $e^{-1}$ half-width of the effective polar diagram calculated from a FCA analysis with SNR larger that $5 \mathrm{~dB}$. Taken from $\mathrm{Yu}$ (2000).

We begin by estimating the scales of the wave oscillations found in the PMSE data revealed in Figs. 1 and 3. First, consider the downward phase propagation discussed above. Although difficult to quantify, we have estimated the rate of the phase propagation to be about $8 \mathrm{~km}$ over a $5 \mathrm{~h}$ period, or roughly $45 \mathrm{~cm} \mathrm{~s}^{-1}$, based on the values of $\mu_{x}$ in Fig. 3 . There is a triple-layer structure in the upper portion of the RTP plot of $\mu_{x}$. The estimate of $45 \mathrm{~cm} \mathrm{~s}^{-1}$ is primarily based on the rate of descent of this structure. The next step is to identify the dominant periodicities in the PMSE data. The period includes both the intrinsic period of the wave motion plus any Doppler shifting of the wave that may occur. The prevailing periodicities in the data were calculated using spectral analysis of the vertical velocity data. First, a 1-min resolution time series was generated by computing the averages of the vertical velocity data over all available heights. The time series was weighted with a Hanning window and its spectrum calculated using a fast Fourier transform. The resulting spectrum contains peaks at frequencies corresponding to periods of $30 \mathrm{~min}$ and $50 \mathrm{~min}$.

Using the periods generated from the spectral analysis and assuming that the observed downward trend in the values of $\mu_{x}$ results solely from wave activity, we can calculate the vertical wavelength of such waves. The periods of $30 \mathrm{~min}$ and $50 \mathrm{~min}$ yield vertical wavelengths of $800 \mathrm{~m}$ and $1300 \mathrm{~m}$, respectively. Similar to the analysis performed to determine the prevailing periods, we have computed a spectrum from an averaged height profile of vertical velocities. The averaged profile was computed for the time interval given by 13:40 to 13:49 UT. The period was chosen to provide a large height range of continuous vertical velocity data. The dominant peaks in the spectrum corresponded to vertical wavelengths of $800 \mathrm{~m}$ and $1200 \mathrm{~m}$. Although this analysis is crude, it shows that some of the observed structures in the PMSE data can be plausibly explained by wave oscillations having vertical wavelengths of $\sim 800-1200 \mathrm{~m}$ and periods of $\sim 30-50 \mathrm{~min}$.

Next, we quantify to what extent the observed PMSE are aspect sensitive. For this, we define the characteristic width $(C W)$ of the brightness distribution function. Following the work of Yu (2000), we show that $C W$, which is just the average of $\sigma_{x}^{\prime}$ and $\sigma_{y}^{\prime}$, can be treated as a proxy for estimations of aspect sensitivity. This is done by relating $C W$ with a more established estimate of aspect sensitivity.

One means of estimating the aspect sensitivity is the "spatial correlation method" from FCA (e.g. Lesicar and Hocking, 1992). Hocking et al. (1987) proposed to parameterize aspect sensitivity by using an estimate of the effective polar diagram of backscattered power given by

$P(\theta)=\exp \left[-\frac{\sin ^{2} \theta}{\sin ^{2} \theta_{s}}-\frac{\sin ^{2} \theta}{\sin ^{2} \theta_{b}}\right]$,

where $\theta$ is the zenith angle, $\theta_{b}$ is the $e^{-1}$ half-width of the radar transmit beam, and $\theta_{s}$ is a measure of the aspect sensitivity. The $e^{-1}$ half-width of the effective polar diagram denoted by $\theta_{s b}$ (degrees) can be found by the equation (e.g. Holdsworth, 1995)

$\theta_{s b}=\frac{15.2 \lambda \sqrt{R_{a x}}}{S_{0.5}}$,

where $\lambda$ is the radar wavelength, and $R_{a x}$ and $S_{0.5}$ are the FCA estimated axis ratio and pattern scale of the diffraction pattern, respectively. Finally, the aspect sensitivity $\theta_{s}$ can be found through the equation

$\sin ^{-2} \theta_{s}=\sin ^{-2} \theta_{s b}-\sin ^{-2} \theta_{b}$.

Since beam effects have not been removed from the brightness distributions used in calculating $\sigma_{x}^{\prime}$ and $\sigma_{y}^{\prime}, C W$ is not directly related to $\theta_{s}$. Therefore, the $e^{-1}$ half-width of the effective polar diagram $\left(\theta_{s b}\right)$ is used for comparison with the characteristic width, rather than $\theta_{s}$. The comparison between $C W$ and $\theta_{s b}$, as given in Yu (2000), is presented in Fig. 4. A linear relationship between the width of the angular brightness and aspect sensitivity is evident.

Given that the half-widths of the brightness distributions are related to the aspect sensitivity, we return our attention to Fig. 3. As mentioned in the previous section, there appears to be a connection between the vertical structuring of SNR and $\sigma_{x}^{\prime}$ and $\sigma_{y}^{\prime}$. In particular, minima in the values of $\sigma_{x}^{\prime}$ and $\sigma_{y}^{\prime}$ seem to correspond with the lower boundaries of the sublayers in the upper regions of the PMSE. A similar relationship was reported for PMSE observations made with the European incoherent scatter (EISCAT) VHF radar. Using frequency-domain interferometry (FDI), Chilson et al. (2001) showed that the lower boundaries of PMSE layers 
correspond to regions of stable scattering layers. The results of Zecha et al. (2001) also show an enhancement of aspect sensitivity on the lower boundary of their PMSE data.

To quantify the dependences between such parameters as SNR, aspect sensitivity, and wave activity, we consider individual height profiles of the relevant quantities. Unfortunately, the lack of continuity in the available data limits our ability to create profiles that are continuous in height for all of the above mentioned parameters. However, we have been able to select three time intervals during the PMSE event for which good height coverage of data could be achieved. The times corresponding to these intervals, which are centered at 12:41, 12:54, and 14:49 UT, are indicated in Figs. 1, 3, and 6 by black vertical lines. Each of the time intervals spans $5 \mathrm{~min}$, a period over which the data have been temporally averaged. The resulting height profiles for the three time intervals are shown in Fig. 5.

Shown in the four left most panels of Fig. 5 are height profiles of parameters which have already been discussed and are shown in Figs. 1 and 3. We note that for all three cases, minima in SNR correspond to maxima in $\sigma_{x}^{\prime}$ and $\sigma_{y}^{\prime}$. The converse is also true; maxima in SNR correspond to minima in $\sigma_{x}^{\prime}$ and $\sigma_{y}^{\prime}$, i.e., SNR is largest in those regions that are the most aspect sensitive. In the neutral atmosphere, aspect sensitive radar echoes often correspond to regions that are statically stable. Reports of the correlation between radar reflectivity and static stability date back to the earliest MST radar observations (see, for example, Gage, 1990). To what extent do we expect static stability to have an impact on our observations? To address this question, we begin by asking whether a correlation exists between the vertical velocity and the characteristic width at scales comparable to the vertical wavelength of the wave oscillations.

It has been shown for the case of the neutral atmosphere that wave perturbations in the vertical velocity are coupled with perturbations in the radar reflectivity (Nastrom and VanZandt, 1994). Nastrom and VanZandt (1994) correlate regions of maximum static stability with regions of waveperturbation induced downward motion. Using similar arguments, Hoppe and Fritts (1995) proposed that downward biases in the vertical velocity observed in the mesosphere result from the tendency of wave-perturbation induced upward motions to be more turbulent.

To test for a correlation between wave-induced vertical atmospheric motion and static stability, we have passed the estimates of vertical velocity and $C W$ through a high-pass Butterworth filter. The filter suppresses oscillations that have vertical wavelengths longer than $4 \mathrm{~km}$, thereby removing large-scale oscillations. Otherwise, the wave-induced oscillations may be obscured by the contributions from the largescale oscillations. The resulting filtered profiles of vertical velocity $W^{\prime}$ and characteristic width $C W^{\prime}$ are shown in the fifth panels from the left of Fig. 5.

We should note that contributions from the horizontal wind can leak into the estimates of the vertical velocity whenever there is a significant tilting of the in-beam refractivity surfaces (Larsen and Röttger, 1991; Palmer et al.,
1991). Therefore, we have corrected for such potential biases in the vertical wind estimates by using the MAOA estimates and the horizontal wind data. The correction was applied before any high-pass filtering.

The height profiles in Fig. 5 reveal a distinctive link between wave-induced perturbations in the vertical velocity $\left(W^{\prime}\right)$ and modulations in the characteristic width of the brightness distribution $\left(C W^{\prime}\right)$. The three time intervals that have been chosen indicate varying levels of correlation between the two parameters. For the time interval centered at 12:41 UT, a clear correlation between $W^{\prime}$ and $C W^{\prime}$ is apparent over all heights. For the next time interval (centered at 12:54 UT), the correlation only exists for the lower portion of the PMSE, i.e., up to a height of about $87 \mathrm{~km}$. Finally, there is no clear correlation between $W^{\prime}$ and $C W^{\prime}$ for the last time interval (centered at 14:49 UT).

Consider those time and height regimes for which the correlation between $W^{\prime}$ and $C W^{\prime}$ exists. Minima in $W^{\prime}$ correspond well with minima in $C W^{\prime}$. If we assume that aspect sensitivity is enhanced through static stability, then we find that the wave-perturbation induced downward motions of air parcels correspond to regions of maximum static stability. This can be understood by recognizing that downward motions in an air parcel correspond to positive changes in its atmospheric density with time. This, in turn, produces conditions of local stability (Mobbs, 1985; Weinstock, 1987). The relationship between $W^{\prime}$ and $C W^{\prime}$ does not seem to hold if the wave must penetrate through a region of elevated vertical shear in the horizontal wind. It appears that in such a case, the stable stratified layers are disrupted by shear-induced turbulence (Gage et al., 1981).

Finally, height profiles of the vertical shear are shown in the right most panels of Fig. 5. These were calculated using

$S_{z}=\sqrt{\left(\frac{\Delta U}{\Delta z}\right)^{2}+\left(\frac{\Delta V}{\Delta z}\right)^{2}}$,

where $\frac{\Delta U}{\Delta z}$ and $\frac{\Delta W}{\Delta z}$ are the vertical gradients in the zonal and meridional wind, respectively. The gradients were calculated using a running, 5-point least-squares fitting algorithm. Therefore, each gradient was calculated over a height interval of $750 \mathrm{~m}$.

We should mention that it is not necessarily the vertical shear of the horizontal wind that is of interest when parameterizing the dynamic stability of flows in the atmosphere, but rather it is the gradient Richardson number, $R i$, which can be calculated through the expression

$R i=\frac{g}{\bar{\theta}} \frac{\Delta \theta}{\Delta z} \frac{1}{S_{z}^{2}}$,

where $g$ is the gravitational acceleration, $\theta$ is the potential temperature, and $\bar{\theta}$ is the mean potential temperature. We have calculated $R i$ using our wind data estimates and calculations of $\theta$ based on the measurements of temperature and density given in Lübken (1999). The resulting $R i$ is shown in Fig. 6, as well as $S_{z}$. 

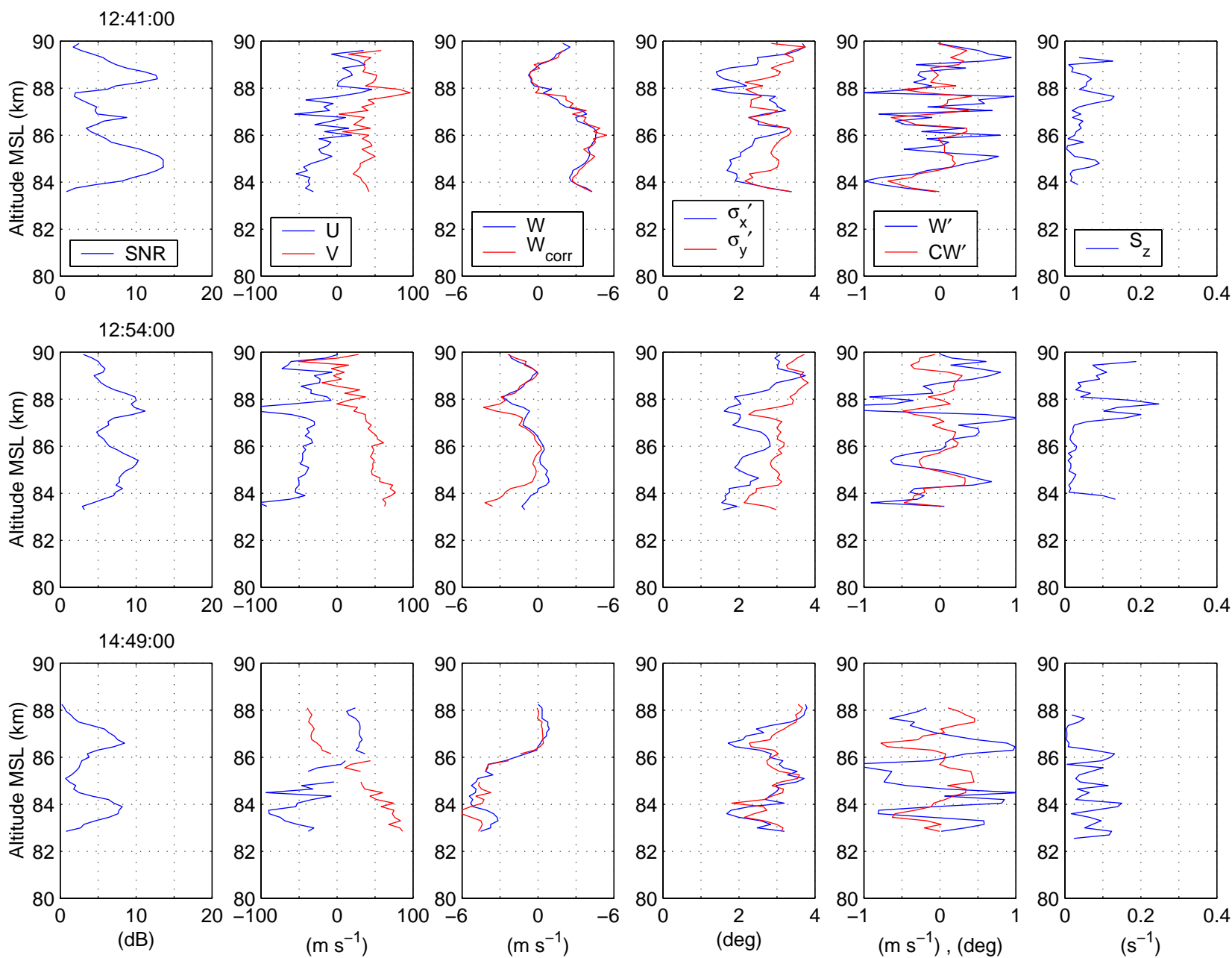

Fig. 5. Height profiles of various observables related to SNR, aspect sensitivity, and wave activity during the PMSE event for three selected time intervals. The four right most panels show estimates of the signal-to-noise ratio (SNR); zonal (U) and meridional (V) components of the horizontal wind; the vertical velocity (W); and the half-widths of the brightness distribution ( $\sigma_{x}^{\prime}$ and $\left.\sigma_{y}^{\prime}\right)$. Height profiles of the high-pass filtered values of the vertical wind $\left(W^{\prime}\right)$ and the high-pass filtered values of the characteristic width of the brightness distribution $\left(C W^{\prime}\right)$ are given in the next column of panels. Estimates of the vertical shear in the horizontal wind $\left(S_{z}\right)$ are shown in the right most panels.

The largest values of $S_{z}$, and correspondingly smallest values of $R i$, are found in the region separating the upper and lower portions of the PMSE layer. The values of $R i$ are, for the most part, smaller than 0.25 within this region. Gradient Richardson numbers less than 0.25 are a necessary, but not sufficient, condition for the creation of dynamic instabilities (Miles and Howard, 1964). One should be very cautious when interpreting the values of $R i$ presented in Fig. 6, since they were calculated from potential temperatures based on seasonally averaged profiles of temperature and density (Lübken, 1999). Nevertheless, the $R i$ data show that vertical shear in the horizontal wind could conceivably lead to dynamic instability during the PMSE event.

\section{Summary and conclusions}

Several features associated with VHF PMSE were already evident from the first observations of the phenomenon. The echoes can be highly structured in the vertical extent; they are often aspect sensitive; and the spectral widths of the Doppler spectra for the echoes are often very narrow. We have demonstrated that these features are possibly related through the interaction of atmospheric wave oscillations within the upper mesosphere and lower thermosphere. Regions of enhanced atmospheric stability can be dynamically induced by upward propagating waves. Wave-induced downward motions in the local position of the PMSE layers correspond to positive changes in the atmospheric density with time. This, in turn, produces conditions of stability, which can be observed as aspect sensitivity. The effect manifests itself in the SNR values of the PMSE as vertical structures which are coupled to the background wave oscillations. The observed downward phase propagations result from the upward propagation of gravity waves, which modulate the background wind.

Another mechanism can be invoked to account for the relationships between SNR and vertical wave motions. It is 

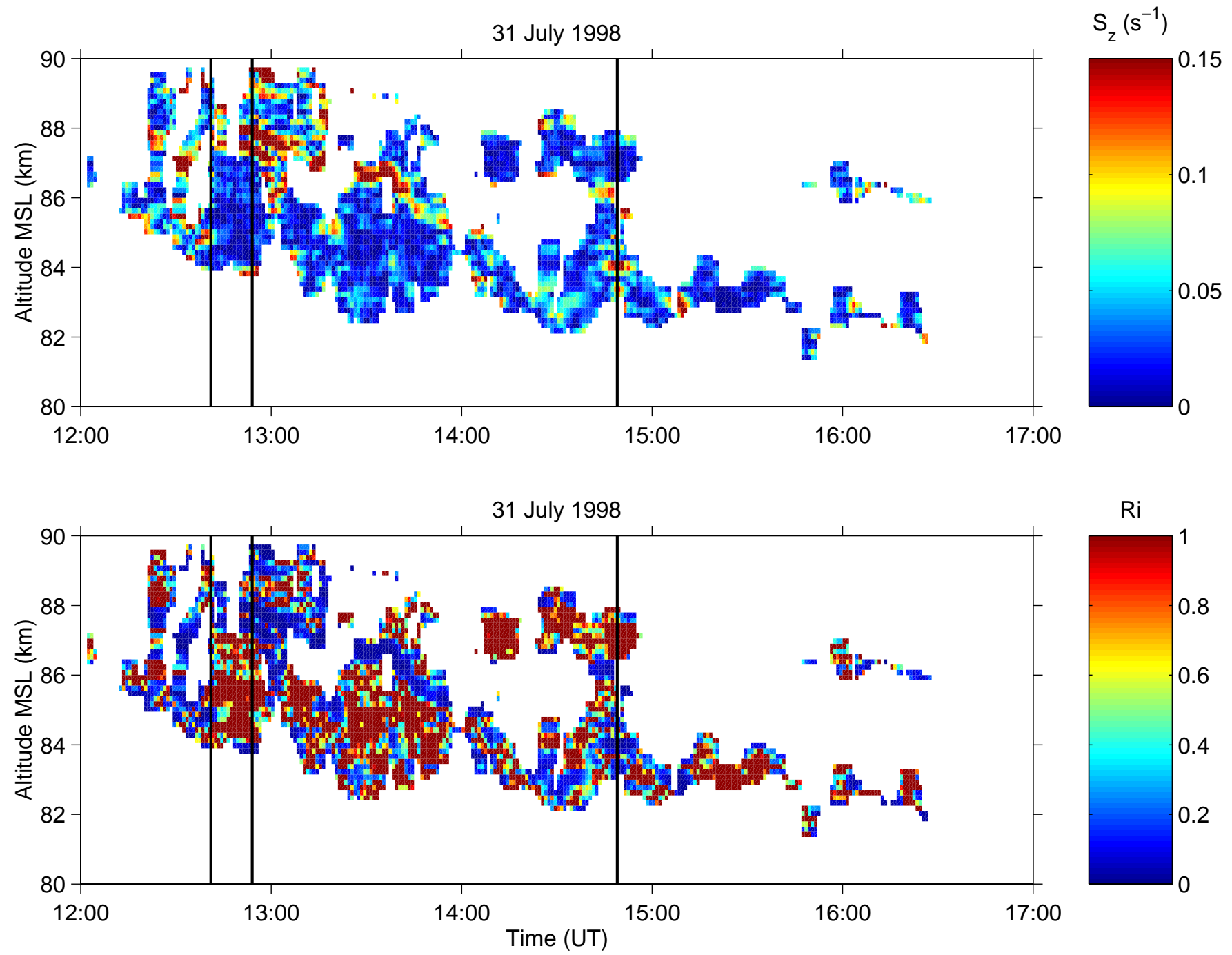

Fig. 6. Range-time-pseudocolor plots of the vertical shear in the horizontal wind $\left(S_{z}\right)$ and the gradient Richardson number $(R i)$.

well established that the occurrence of PMSE is linked to the cold temperatures of the polar summer mesopause region. The cold temperatures are needed for the charged ice aerosols that lead to the production of PMSE. Adiabatic cooling and warming resulting from the vertical displacements of air parcels lead to localized variations in the temperature field. These, in turn, influence the growth and decay processes of the ice particles. The observed phase relation between the PMSE SNR and the wave-induced perturbations in the vertical velocity were attributed to temperature fluctuations by Cho and Morley (1995) and Rüster (1995). This would indicate that fluctuations in the vertical velocity lead to the fluctuations in temperature due to adiabatic cooling and heating. If SNR is directly related to the size and number of ice particles, which are assumed to be directly related to the temperature, then vertical velocity fluctuations should lead SNR fluctuations by $90^{\circ}$. The primary problem with the proposal is that the time scales for the production and destruction of ice particles are often longer than the periods of the wave motions.

These observations, similar to previous observations of PMSE, reinforce the need for more of a better fundamental understanding of radar scattering/reflection processes. Given the difficulty of achieving in situ measurements of PMSE from rocket-borne platforms, we are motivated to pursue additional radar imaging investigations of this phenomenon. The present study, together with that of $\mathrm{Yu}$ et al. (2001), demonstrate the utility of CRI applications for the study of PMSE. In particular, CRI can successfully reveal structures and wave-induced perturbations in the the radar reflectivity, as revealed through maps of the brightness distribution. These have been used in the present study to quantify inbeam aspect sensitive for PMSE and to estimate the MAOA of the bulk backscattered signals. We encourage additional observations of PMSE using radar imaging.

Acknowledgements. P. B. C. was supported by the Environment and Space Research Institute (MRI) in Kiruna during portions of this work. R. D. P. and T. Y. Y. were supported by the Division of Atmo- 
spheric Sciences of the National Science Foundation through grant ATM 99-08616. S. K. is financed by the Swedish Natural Science Research Council (NFR) and MRI. ESRAD is funded jointly by the Swedish Space Corporation and the Swedish Natural Science Research Council.

Topical Editor D. Murtagh thanks two referees for their help in evaluating this paper.

\section{References}

Balsley, B. B., Woodman, R. F., Sarango, M., Rodriguez, R., Urbina, J., Ragaini, E., Carey, J., Huaman, M., and Giraldez, A.: On the lack of southern hemisphere polar mesosphere summer echoes, J. Geophys. Res., 100, 11 685-11 693, 1995.

Blix, T. A.: Small scale plasma and charged aerosol variations and their importance for polar mesosphere summer echoes, Adv. Space Res., 24, 537-546, 1999.

Briggs, B. H.: The analysis of spaced sensor records by correlation techniques, in: Handbook for MAP, (Ed) Vincent, R. A., 13, 823-833, SCOSTEP Secr., Univ. of Ill., Urbana, 1984.

Chau, J. L. and Woodman, R. F.: Three-dimensional coherent radar imaging at Jicamarca: Comparison of different inversion techniques, J. Atmos. Sol. Terr. Phys., 63, 253-261, 2001.

Chilson, P. B., Czechowsky, P., Klostermeyer, J., Rüster, R., and Schmidt, G.: An investigation of measured temperature profiles and VHF mesosphere summer echoes at midlatitudes, J. Geophys. Res., 102, 23 819-23 828, 1997.

Chilson, P. B., Kirkwood, S., and Nilsson, A.: The Erange MST radar: A brief introduction and procedure for range validation using balloons, Radio Sci., 34, 427-436, 1999.

Chilson, P. B., Kirkwood, S., and Häggström, I.: Frequency-domain interferometry mode observations of PMSE using the EISCAT VHF radar, Ann. Geophysicae, 18, 1599-1612, 2001.

Cho, J. Y. N. and Kelley, M. C.: Polar mesosphere summer radar echoes: Observations and current theories, Rev. Geophys. Space Phys., 31, 243-265, 1993.

Cho, J. Y. N. and Morley, R. L.: PMSE dependence on long-period vertical motions, Geophys. Res. Lett., 22, 1197-1200, 1995.

Cho, J. Y. N. and Röttger, J.: An updated review of polar mesosphere summer echoes: Observation, theory, and their relationship to noctilucent clouds and subvisible aerosols, J. Geophys. Res., 102, 2001-2020, 1997.

Cho, J. Y. N., Hall, T. M., and Kelley, M. C.: On the role of charged aerosols in polar mesosphere summer echoes, J. Geophys. Res., 97, 875-886, 1992.

Czechowsky, P. and Rüster, R.: VHF radar observations of turbulent structures in the polar mesopause region, Ann. Geophysicae, 15, 1028-1036, 1997.

Czechowsky, P., Reid, I. M., and Rüster, R.: VHF radar measurements of the aspect sensitivity of the summer polar mesopause over Andenes $\left(69^{\circ} \mathrm{N}, 16^{\circ} \mathrm{E}\right)$ Norway, Geophys. Res. Lett., 15, 1259-1262, 1988.

Ecklund, W. L. and Balsley, B. B.: Long-term observations of the arctic mesopause with the radar at Poker Flat, Alaska, J. Geophys. Res., 86, 7775-7780, 1981.

Fritts, D. C., Smith, S. A., Balsley, B. B., and Philbrick, C. R.: Evidence of gravity wave saturation and local turbulence production in the summer mesosphere and lower thermosphere during the STATE experiment, J. Geophys. Res., 93, 7015-7025, 1988.

Gage, K. S.: Radar observations of the free atmosphere: Structure and dynamics, in: Radar in Meteorology, (Ed) Atlas, D., pp. 534-565, Am. Meteorol. Soc., Boston, Mass., 1990.

Gage, K. S. and Green, J. L.: Evidence for specular reflection from monostatic VHF radar observations of the stratosphere, Radio Sci., 13, 991-1001, 1978.

Gage, K. S., Carter, D. A., and Ecklund, W. L.: The effect of gravity waves on specular echoes observed by the Poker Flat MST radar, Geophys. Res. Lett., 8, 599-602, 1981.

Haykin, S., Justice, J. H., Owsley, N. L., Yen, J. Y., and Kak, A. C.: Array Signal Processing, Prentice-Hall Inc., Englewood Cliffs, NJ, 1985.

Hocking, W. K.: Radar studies of small scale structure in the upper middle atmosphere and lower ionosphere, Adv. Space Res., 7, 327-338, 1987.

Hocking, W. K. and Röttger, J.: Studies of polar mesosphere summer echoes over EISCAT using calibrated signal strengths and statistical parameters, Radio Sci., 32, 1425-1444, 1997.

Hocking, W. K., Rüster, R., and Czechowsky, P.: Absolute reflectivities and aspect sensitivities of VHF radio wave scatterers measured with the SOUSY radar, Radio Sci., 48, 131-144, 1987.

Hocking, W. K., May, P. T., and Röttger, J.: Interpretation, reliability and accuracies of parameters deduced by the spaced antenna method in middle atmosphere applications, Pure Appl. Geophys., 130, 571-604, 1989.

Holdsworth, D. A.: Signal analysis with applications to atmospheric radars, Ph.D. thesis, University of Adelaide, 1995.

Hooper, D. A. and Thomas, L.: The small-scale structure of VHF mesospheric summer echo layers observed at mid-latitudes, Ann. Geophysicae, 15, 1037-1047, 1997.

Hoppe, U.-P. and Fritts, D. C.: High-resolution measurements of vertical velocity with the European incoherent scatter VHF radar 1. Motion field characteristics and measurement biases, J. Geophys. Res., 100, 16813-16825, 1995.

Huaman, M. M. and Balsley, B. B.: Long-term-mean aspect sensitivity of PMSE determined from the Poker Flat MST radar data, Geophys. Res. Lett., 7, 947-950, 1998.

Hysell, D. L.: Radar imaging of equatorial F region irregularities with maximum entropy interferometry, Radio Sci., 31, 15671578, 1996.

Hysell, D. L. and Woodman, R. F.: Imaging coherent backscatter radar observations of topside equatorial spread F, Radio Sci., 32, 2309-2320, 1997.

Kirkwood, S. and Réchou, A.: Planetary-wave modulations of PMSE, Geophys. Res. Lett., 25, 4509-4512, 1998.

Kudeki, E. and Sürücü, F.: Radar interferometric imaging of fieldaligned plasma irregularities in the equatorial electrojet, Geophys. Res. Lett., 18, 41-44, 1991.

Larsen, M. F. and Röttger, J.: VHF radar measurements of inbeam incidence angles and associated vertical-beam radial velocity corrections, J. Atmos. Ocean. Tech., 8, 477-490, 1991.

Lesicar, D. and Hocking, W. K.: Studies of seasonal behaviour of the mesospheric scatterers using a $1.98 \mathrm{MHz}$ radar, J. Atmos. Terr. Phys., 54, 295-309, 1992.

Lübken, F.-J.: Thermal structure of the Arctic summer mesopause, J. Geophys. Res., 104, 9135-9150, 1999.

Miles, J. W. and Howard, L. N.: Note on a heterogeneous shear flow, Q. J. R. Meteorol. Soc., 20, 331-336, 1964.

Mobbs, S. D.: Propagation of nonlinear internal gravity waves at stratospheric and mesospheric heights. Part iii: The wave shape, Ann. Geophysicae, 3, 599-608, 1985.

Nastrom, G. D. and VanZandt, T. E.: Mean vertical motions seen by radar wind profilers, J. Appl. Meteorol., 33, 984-995, 1994.

Nussbaumer, V., Fricke, K. H., Langer, M., Singer, W., and von 
Zahn, U.: First simultaneous and common volume observations of noctilucent clouds and polar mesosphere summer echoes by lidar and radar, J. Geophys. Res., 101, 19 161-19 167, 1996.

Palmer, R. D., Larsen, M. F., Woodman, R. F., Fukao, S., Yamamoto, M., Tsuda, T., and Kato, S.: VHF radar interferometry measurements of vertical velocity and the effect of tilted refractivity surfaces on standard Doppler measurements, Radio Sci., 26, 417-427, 1991.

Palmer, R. D., Gopalam, S., Yu, T.-Y., and Fukao, S.: Coherent radar imaging using Capon's method, Radio Sci., 33, 1585-1589, 1998.

Palmer, R. D., Yu, T.-Y., and Chilson, P. B.: Range imaging using frequency diversity, Radio Sci., 34, 1485-1496, 1999.

Reid, I. M., Czechowsky, P., Rüster, R., and Schmidt, G.: First VHF radar measurements of mesopause summer echoes at midlatitudes, Geophys. Res. Lett., 16, 135-138, 1989.

Röttger, J. and Vincent, R. A.: VHF radar studies of tropospheric velocities and irregularities using spaced antenna techniques, Geophys. Res. Lett., 5, 917-920, 1978.

Röttger, J., Hoz, C. L., Kelley, M. C., Hoppe, U.-P., and Hall, C.: The structure and dynamics of polar mesosphere summer echoes observed with the EISCAT $224 \mathrm{MHz}$ radar, Geophys. Res. Lett., 15, 1353-1356, 1988.

Röttger, J., Hoz, C. L., Franke, S. J., and Liu, C. H.: Steepening of reflectivity structures detected in high-resolution Doppler spectra of polar mesosphere summer echoes (PMSE) observed with the EISCAT 224-MHz radar, J. Atmos. Terr. Phys., 52, 939-954, 1990.

Rüster, R.: Velocity and associated echo power variations in the summer polar mesosphere, Geophys. Res. Lett., 22, 65-67, 1995.

Stebel, K., Barabash, V., Kirkwood, S., Siebert, J., and Fricke, K.H.: Polar mesosphere summer echoes and noctilucent clouds:
Simultaneous and common-volume observations by radar, lidar and CCD camera, Geophys. Res. Lett., 27, 661-664, 2000.

Thomas, L., Astin, I., and Prichard, T.: The characteristics of VHF echoes from the summer mesopause region at mid-latitudes, J. Atmos. Terr. Phys., 54, 969-977, 1992.

Thompson, A. R.: Interferometry and Synthesis in Radio Astronomy, John Wiley, New York, 1986.

von Zahn, U. and Bremer, J.: Simultaneous and common-volume observations of noctilucent clouds and polar mesosphere summer echoes, Geophys. Res. Lett., 26, 1521-1524, 1999.

Weinstock, J.: The turbulence field generated by a linear gravity wave, J. Atmos. Sci., 44, 410-420, 1987.

Woodman, R. F.: Coherent radar imaging: Signal processing and statistical properties, Radio Sci., 32, 2372-2391, 1997.

Woodman, R. F. and Chu, Y.-H.: Aspect sensitivity measurements of VHF backscatter made with the Chung-Li radar: Plausible mechanisms, Radio Sci., 24, 113-125, 1989.

Woodman, R. F., Balsley, B. B., Aquino, F., Flores, L., Vazquez, E., Sarango, M., Huaman, M. M., and Soldi, H.: First observations of polar mesosphere summer echoes in antarctica, J. Geophys. Res., 104, 22 577-22 590, 1999.

Yu, T.-Y.: Radar studies of the atmosphere using spatial and frequency diversity, Ph.D. thesis, University of Nebraska-Lincoln, 2000.

Yu, T.-Y., Palmer, R. D., and Hysell, D. L.: A simulation of study of coherent radar imaging, Radio Sci., 35, 1129-1141, 2000.

Yu, T.-Y., Palmer, R. D., and Chilson, P. B.: An investigation of scattering mechanisms and dynamics in PMSE using coherent radar imaging, J. Atmos. Sol. Terr. Phys., 63, 1797-1810, 2001.

Zecha, M., Röttger, J., Singer, W., Hoffmann, P., and Keuer, D.: Scattering properties of PMSE irregularities and refinements of velocity estimates, J. Atmos. Sol. Terr. Phys., 63, 201-214, 2001. 